\title{
Observation of extreme nonreciprocal wave amplification from single soliton-soliton collisions
}

\author{
Feifei Xin $\odot,{ }^{1,2}$ Mariano Flammini, ${ }^{1}$ Fabrizio Di Mei, ${ }^{1}$ Ludovica Falsi, ${ }^{1}$ Davide Pierangeli, ${ }^{1,3}$ \\ Aharon J. Agranat, ${ }^{4}$ and Eugenio DelRe ${ }^{1,5}$ \\ ${ }^{1}$ Dipartimento di Fisica, Università di Roma La Sapienza, 00185 Rome, Italy \\ ${ }^{2}$ College of Physics and Materials Science, Tianjin Normal University, 300387, Tianjin, China \\ ${ }^{3}$ ICL-2DMOST, College of Optoelectronic Engineering, Shenzhen University, 518060 Shenzhen, China \\ ${ }^{4}$ The Brojde Center for Innovative Engineering and Computer Science, The Hebrew University, Jerusalem 91904, Israel \\ ${ }^{5}$ ISC-CNR, Università di Roma La Sapienza, 00185 Rome, Italy
}

(Received 27 April 2019; revised manuscript received 10 July 2019; published 11 October 2019)

\begin{abstract}
We report the observation of strong nonreciprocal soliton amplification mediated by a Raman-scattering-like effect in single isolated collisions. A pump soliton is found to lose the greater part of its energy to a signal soliton, irrespective of the pump-signal relative amplitude. The result is an efficient rectifying mechanism able to accumulate energy into extreme waves. Experiments are carried out through photorefractive soliton twowave mixing with a gain coefficient of up to $80 \mathrm{~cm}^{-1}$ that emerges in conditions of nonlinear response, leading to the formation of rogue waves.
\end{abstract}

DOI: 10.1103/PhysRevA.100.043816

\section{INTRODUCTION}

Long-tailed statistics and extreme phenomena observed in many different physical systems still lack a general explanatory picture $[1,2]$. One established fact is that a microscopic nonreciprocal rectifying mechanism can cause anomalous fluctuations and ultimately bring about macroscopic, statistically improbable, events. This is well known for thermally agitated systems, where rectification is the driving mechanism of Brownian motors $[3,4]$. Rogue waves (RWs) are localized extreme perturbations with impossibly high peak intensities and an associated long-tailed probability distribution [5-12]. It follows that a possible breakthrough in the understanding of RWs is the identification of an underlying dominant nonreciprocal energy-exchange mechanism that acts to rectify fluctuations. In optical fibers and in photorefractive (PR) crystals, RWs form in time and in space, respectively, in conditions where wave propagation is dominated by interacting solitons [13-15]. While soliton collisions are elastic in a system obeying an integrable model, mixed systems [16] and nonintegrable corrections allow energy and momentum exchange $[17,18]$. This has been widely studied in open systems, such as for pulses propagating in externally pumped erbium-doped fibers [19,20], and continuous-wave ytterbium-doped fiber lasers [21]. For systems with no distributed amplification, typical of soliton and RW studies, local corrections, such as higher-order Kerr effects associated to saturation, are reciprocal and do not allow microscopic rectification. Nonreciprocal energy exchange between waves is mediated by the leading nonlocal nonlinear correction associated to Raman scattering (RS) in fibers [22-25] and its equivalent in space, i.e., diffusion-driven two-wave mixing (TWM) [26,27]. Global nonreciprocity emerges as a consequence of time nonlocality paired with causality in fibers and, equivalenty, to the space nonlocality paired with the direction of an external bias field. Previous studies into the effects of stimulated RS in solitonsoliton collisions have shown theoretically and experimentally how a weaker soliton can feed energy to a more intense one $[28,29]$. Theoretical studies have generalized the picture to also include a limited soliton-soliton energy transfer [30] but still, to date, no experimental evidence of a rectifying mechanism in soliton-soliton collisions has been reported.

In this paper, we investigate soliton-soliton collisions in PR crystals in conditions that can lead to RW formation [14]. In our experiments, we find that even single isolated coherent soliton-soliton collisions are dominated by strong nonreciprocal energy transfer. The direction of this energy transfer is determined by the external bias electric field, irrespective of the amplitudes of the two interacting solitons.

\section{MODEL}

We consider light propagating in a biased crystal heated above the Curie temperature $T>T_{C}$, where anomalous dielectric response and structural metastability leads to a strong electro-optic effect [31,32] with modulational instability, spatial solitons, and optical turbulence [14,33,34]. The system can be discussed in the simplified paraxial one-plus-onedimensional $(1+1 \mathrm{D})$ case, that is, for beams that propagate along one axis, say the $z$ axis, while they diffract and suffer self-focusing only in one transverse direction (the $x$ axis). The slowly varying amplitude $A$ of the optical field obeys the generalized nonlinear Schrodinger equation,

$$
i \partial_{z} A=-\frac{1}{2 k} \partial_{x x}^{2} A+\frac{k}{n_{0}} \Delta n(\bar{I}) A,
$$

where $k=2 \pi n_{0} / \lambda, n_{0}$ is the unperturbed crystal index of refraction, $\lambda$ is the optical wavelength, and $\bar{I}=|A|^{2} / I_{b}$ is the optical intensity normalized to the homogeneous background illumination. The quadratic electro-optic effect leads to a 
(a)
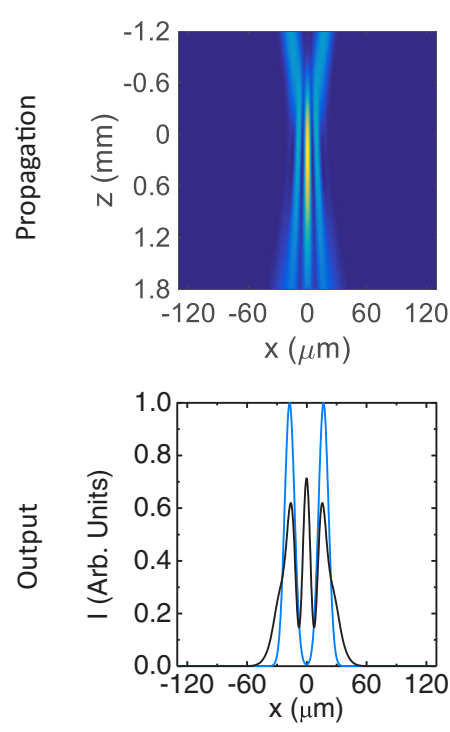

(b)
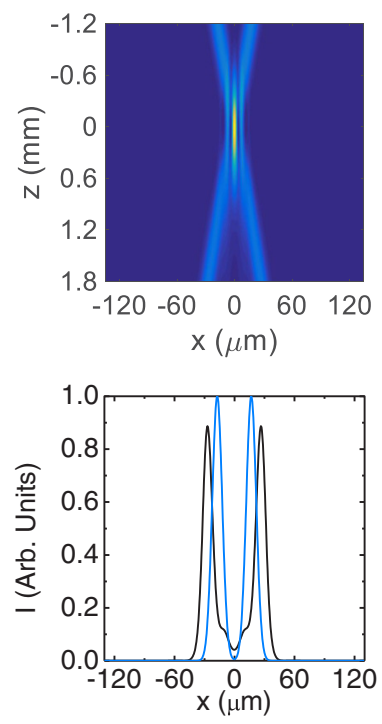

(c)

$\operatorname{Raman}\left(t_{d}>0\right)$
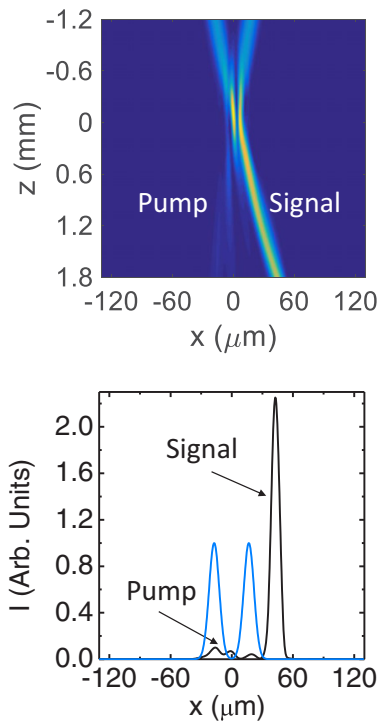

(d) $\quad$ Raman $\left(t_{d}<0\right)$
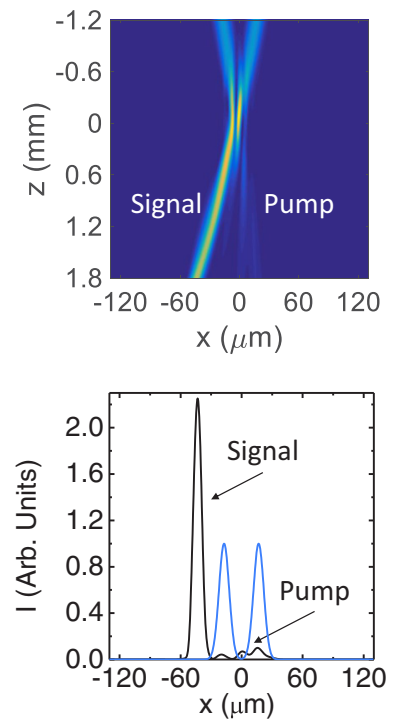

FIG. 1. Strong nonreciprocal energy transfer in a soliton collision mediated by an RS-like correction. Top row: Top-view intensity along propagation; second row: input (blue line) versus output (black line) transverse intensity profile. (a) Linear propagation for mutually coherent beams. (b) Nonlinear Kerr propagation in a model of negligible RS, compared to (c) and (d) where RS plays an important role.

nonlinear response $\Delta n(\bar{I})$ that reads $[35,36]$

$$
\Delta n(\bar{I})=\Delta n_{0}\left[\frac{1}{(1+\bar{I})^{2}}+2 a \frac{\partial_{x} \bar{I}}{(1+\bar{I})^{2}}+a^{2}\left(\frac{\partial_{x} \bar{I}}{1+\bar{I}}\right)^{2}\right],
$$

where $\Delta n_{0}=(1 / 2) n_{0}^{3} g_{\text {eff }} \varepsilon_{0}^{2} \chi^{2} E_{0}^{2}$, with $g_{\text {eff }}$ the effective quadratic electro-optic coefficient, $\varepsilon_{0}$ the vacuum dielectric constant, $\chi=\epsilon_{r}-1$ the low-frequency susceptibility, $E_{0}$ the bias electric field applied in the transverse $x$ direction, and $a=k_{B} T /\left(q E_{0}\right)$, where $k_{B}$ is the Boltzmann constant, $T$ the sample temperature, and $q$ the electron charge. The physical underpinnings involved are identified casting Eq. (2) in the dimensionless form

$$
i \partial_{\zeta} u+\frac{1}{2} \partial_{\xi \xi}^{2} u-\frac{1}{2} \frac{u}{\left(1+|u|^{2}\right)^{2}}=t_{d} \frac{\partial_{\xi}|u|^{2}}{\left(1+|u|^{2}\right)^{2}} u,
$$

with $\quad u=A / \sqrt{I_{b}}, \quad|u|^{2}=\bar{I}, \quad \zeta=2 k \Delta n_{0} z / n_{0}, \quad \xi=$ $k x \sqrt{2 \Delta n_{0} / n_{0}}, t_{d}=a k \sqrt{2 \Delta n_{0} / n_{0}}$, and the third term in Eq. (2), mediated solely by thermal charge diffusion, is neglected [37]. This reduces to the Raman-modified nonlinear Schrodinger equation (NLSE) valid for conventional Kerr-like solitons in optical fibers in the unsaturated limit $\left(|u|^{2} \ll 1\right)$ [22]:

$$
i \partial_{\zeta} u+\frac{1}{2} \partial_{\xi \xi}^{2} u+|u|^{2} u=t_{d} \partial_{\xi}|u|^{2} u .
$$

The first term in Eq. (2), mediated by the PR screening of the external bias field caused by photoinduced charge drift, leads to the third term on the left-hand side of Eqs. (3) and (4) and acts formally as Kerr self-focusing [36]. In turn, the second term in Eq. (2), mediated by the coupling of charge drift with charge thermal diffusion, leads to the term on the right-hand side of Eqs. (3) and (4) and formally constitutes RS. In PR crystals, both self-focusing and RS are caused by indirect steady-state photoinduced space-charge effects that have the same leading macroscopic nonlinear model of their counterparts encountered in fiber pulse propagation, while the microscopic origin is different in the two cases. Understandably, the unsaturated limit of Eq. (4) will be superseded by the full saturated version of the model in Eq. (3) in conditions leading to extreme wave amplitudes.

The presence of a RS-like mechanism in Eq. (3) fundamentally alters the integrable NLSE in that it allows coherent energy exchange, or TWM, between solitons [38]. However, in distinction to other effects that break integrability, such as saturation in the response [39], here the transverse inversion symmetry $\xi \rightarrow-\xi$ is broken, and this introduces nonreciprocity in dynamics [26]. For example, in optical fiber, this broken symmetry is associated to causality in the response $\left(t_{d}>0\right)$ [22], so RS is predicted to transfer energy

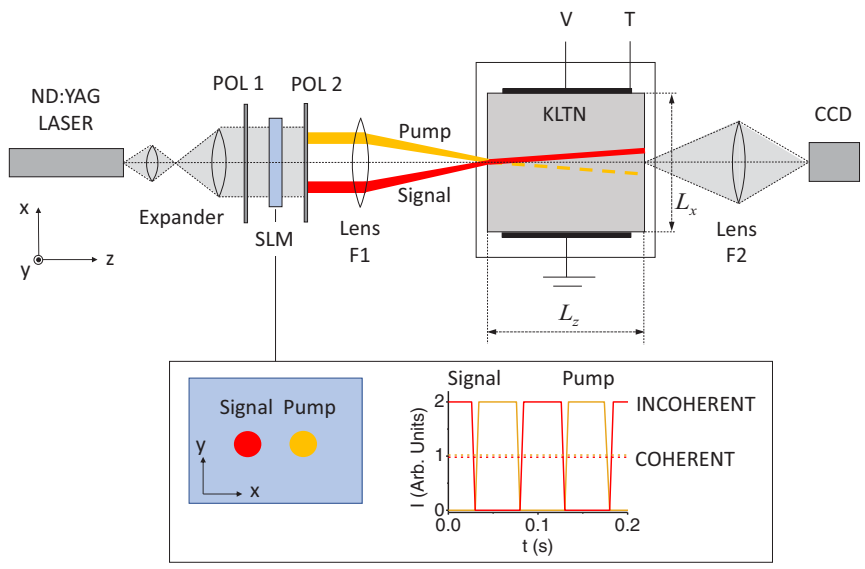

FIG. 2. Experimental setup. The signal and pump beams are prepared in the transverse spatial wave-vector space using an intensity SLM. The inset shows temporal modulation of input signal and pump light that allows the study of incoherent collisions. 
to slower red-shifted giant-amplitude solitons $[5,13]$. In our present case, the nonreciprocity is associated to the sign of the external bias field $E_{0}$, that determines the sign of $a$, and hence $t_{d}$, in Eq. (3).

In Fig. 1, we show soliton TWM through a beampropagation-method integration of Eq. (3) in the conditions investigated experimentally (see below). Energy exchange is associated to the RS-like correction (second versus third and fourth columns) and occurs efficiently only for coherent collisions, while for incoherent collisions negligible energy transfer is found in all cases (see Appendix). The sign of $t_{d}$ is determined by the relative orientation of the external bias field $E_{0}$, for the specific photoinduced dominant ion charge $q$ (electrons in our specific experiments), that then determines the direction of the nonreciprocal energy transfer (third and fourth columns) [40]. Note in Figs. 1(c) and 1(d) how the solitons also self-bend during propagation in a direction fixed by $t_{d}$, a phenomenon associated to the RS-like correc- tion that naturally leads to a redshift in the pulse spectrum $[22,28,41,42]$.

\section{EXPERIMENT}

Experiments are carried out in a compositionally disordered PR KLTN crystal (potassium-lithium-Ttantalateniobate $-\mathrm{K}_{0.99} \mathrm{Li}_{0.01} \mathrm{Ta}_{0.60} \mathrm{Nb}_{0.40} \mathrm{O}_{3}$ ). The crystal was grown through the top-seeded solution method by extracting a zerocut optical quality specimen that measures $L_{x}=2.6 \mathrm{~mm}, L_{y}=$ $3.4 \mathrm{~mm}, L_{z}=1.8 \mathrm{~mm}$ along the $x-y-z$ axes. It has an $n_{0}=2.3$ and a $g_{\text {eff }}=0.14 \mathrm{~m}^{4} \mathrm{C}^{-2}$ when the optical polarization is parallel to one principal axis, say the $x$ axis, also parallel to the external bias field $\mathbf{E}_{0}=E_{0} \mathbf{u}_{x}$. The sample has a temperaturedependent quasistatic susceptibility: Operating at a temperature close to the Curie point, $T=T_{C}+6 \mathrm{~K}$, we achieve a high $\epsilon_{r} \simeq 1.5 \times 10^{4}$ that greatly enhances the electro-optic response. Soliton collisions are observed using the setup
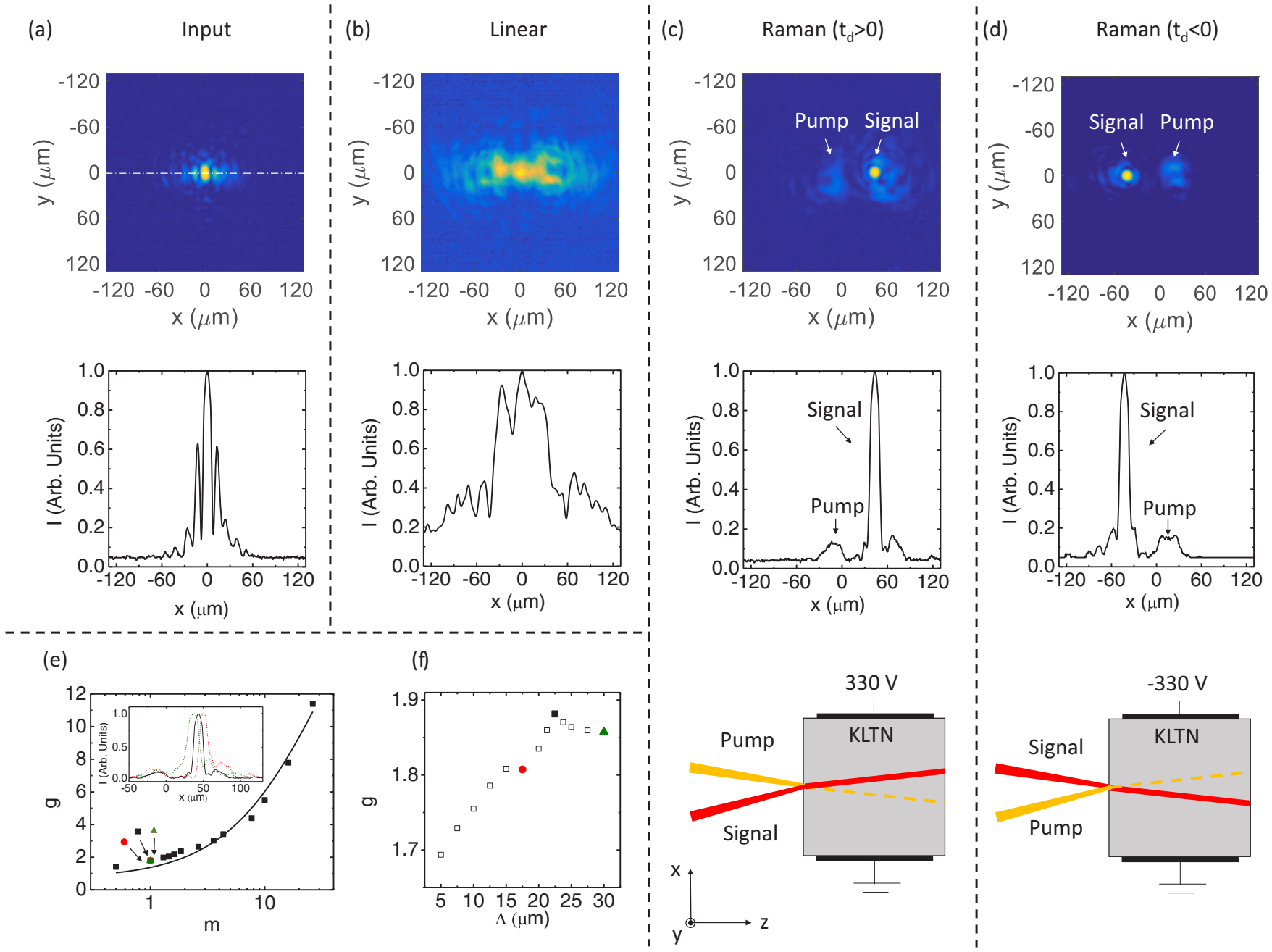

FIG. 3. Extreme wave amplification through nonreciprocal energy transfer in a single soliton-soliton collision. (a) Input intensity distribution (top) and profile (bottom); (b) output intensity distribution and profile for linear propagation $(V=0)$. (c) Strong directional energy transfer for $t_{d}>0$ and (d) $t_{d}<0$. (e) TWM gain $g$ versus input pump-signal $m$ intensity ratio for a collision angle of $\theta=12$ mrad (black squares). Red dot and green triangle are for $\theta=15 \mathrm{mrad}$ and $\theta=9 \mathrm{mrad}$, respectively. The continuous line is the analytical prediction from Eq. (5). Inset: Transverse intensity $x$ profiles for $m=1$ and $\theta=12 \mathrm{mrad}$ (black line), $\theta=15 \mathrm{mrad}$ (red dashed line), $\theta=9 \mathrm{mrad}$ (green dashed line). (f) TWM gain $g$ versus interference grating period $\Lambda$ for different inspected angles (red dot for $\theta=15 \mathrm{mrad}$, black square for $\theta=12 \mathrm{mrad}$, and green triangle for $\theta=9 \mathrm{mrad}$.) compared to those predicted (white squares) from numerical simulations. 
(a) Input

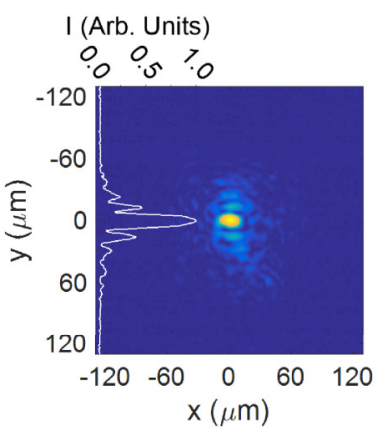

(b) Linear

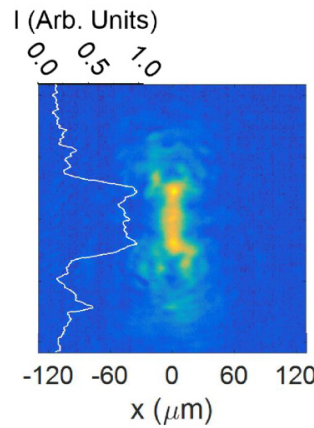

(c) Raman $\left(t_{d}>0\right)$

(d) $\operatorname{Raman}\left(\mathrm{t}_{\mathrm{d}}<0\right)$

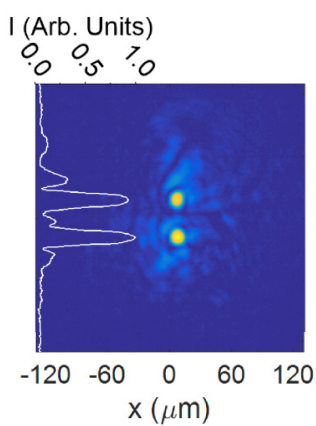

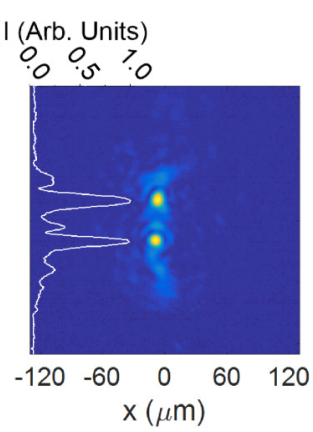

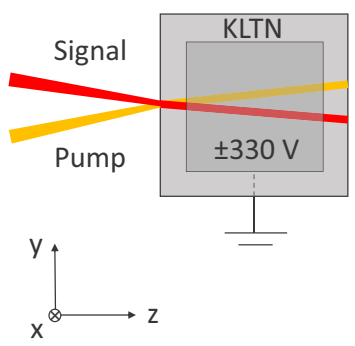

FIG. 4. Absence of TWM when signal and pump solitons are physically not distinguished. The input beams (a) cross inside the sample in the $y$ direction and diffract for a linear propagation $(V=0)(\mathrm{b})$, leading to soliton formation with no nonreciprocal energy transfer for $t_{d}>0$ (c) and $t_{d}<0$ (d).

illustrated in Fig. 2. A $\lambda=532 \mathrm{~nm}$ continuous-wave $110 \mathrm{~mW}$ beam from a $\mathrm{Nd}$ :YAG laser is first expanded and made to propagate through a liquid-crystal spatial-light-modulator (SLM), sandwiched in between two crossed polarizers (POL1 and POL2). A focusing lens $(\mathrm{F} 1=60 \mathrm{~mm})$ is used to image the transmitted light onto the input facet of the sample, with its principal axes along the $x, y, z$ directions, generating the two colliding signal $\left(I_{s}\right)$ and pump $\left(I_{p}\right)$ beams. A voltage $V$ is delivered to the opposite $y z$ facets, so $E_{0}=V / L_{x}$. Propagation along the $z$ axis, through the whole length of the sample $L_{z}$, is analyzed, imaging the input and output facets through a second lens $(\mathrm{F} 2=50 \mathrm{~mm})$ onto a CCD camera. For the study of coherent collisions, both beams are transmitted by the SLM simultaneously (see the red and yellow illustrations in the inset of Fig. 2). For the study of incoherent collisions, each of the beams is alternatively switched on (with twice the intensity of the coherent experiment) and off so that at each instant in time only the signal or the pump are propagating through the sample. This amounts to an incoherent collision when the switching time $(0.05 \mathrm{~s})$ is much smaller than the characteristic response time of the nonlinearity (100 $\mathrm{s}$, in our case).

Results are reported in Fig. 3. The angle between the two crossing beams is $\theta=12 \mathrm{mrad}$ (inside the sample) with their polarization parallel to the $x$ axis. The beams have the same power $(1 \mu \mathrm{W})$, while the background illumination is $5 \%$ of each beam's peak intensity. When the two beams are coherent, for linear propagation (i.e., for $V=0$ ), each $10-\mu \mathrm{m}$ FWHM beam at the input [Fig. 3(a)] spreads at the output to $45 \mu \mathrm{m}$ [Fig. 3(b)]. A characteristic interference pattern forms (with a period $12 \mu \mathrm{m}$ at input and $34 \mu \mathrm{m}$ at output). For a $V=330 \mathrm{~V}\left(t_{d}>0\right)$, a large portion of the energy from one soliton is transferred to the other, even though the two beams are only crossing for several hundred micrometers [Fig. 3 (c)]. Nonreciprocity is demonstrated by inverting the underlying asymmetry in the RS-like response, associated to the direction of the bias electric field. Congruently, for $V=$ $-330 \mathrm{~V}$ (i.e., $t_{d}<0$ ), the same effect takes place but in the opposite direction [Fig. 3(d)]. The role of coherence is tested having the very same experiment take place but in conditions in which the two diffracting beams, and hence the resulting solitons, are mutually incoherent (see Appendix). Here the two solitons pass through each other with negligible exchange of energy and negligible dependence on the direction of the external bias.

A signature of TWM is that the nonreciprocal amplification of the signal obeys the relationship [26]

$$
g=\frac{I_{s}\left(L_{z}\right)}{I_{s}(0)}=\frac{1+m}{1+m e^{-\gamma L_{i}}} e^{-\alpha L_{z}},
$$

where $L_{i}$ is the interaction length and $m=I_{p}(0) / I_{s}(0)$ is the input pump-signal intensity ratio, $\gamma$ is the TWM gain coefficient, $\alpha$ is the absorption coefficient, and $L_{z}$ is the length of the sample. In Fig. 3(e), we report the measured soliton peak signal output-to-input intensity ratio $g$ for different values of $m$ (measured for peak input intensities) and the best fit to (a)
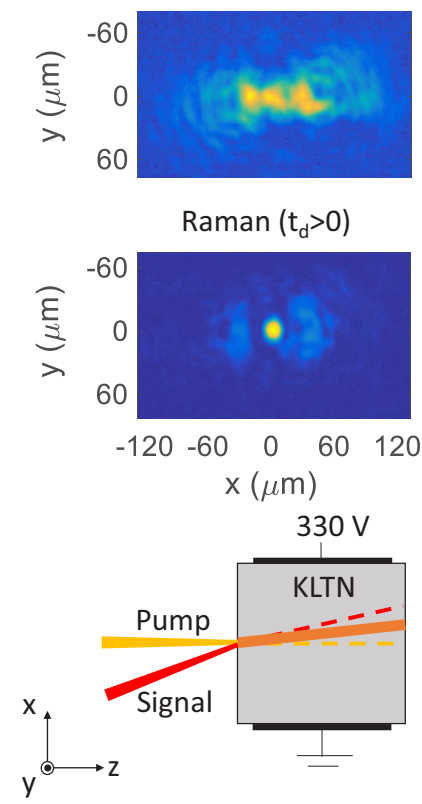

(b)
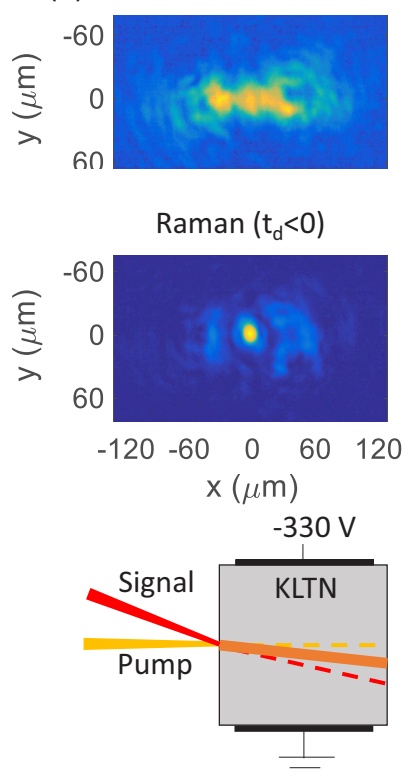

FIG. 5. Soliton TWM and reciprocal fusion. For a colliding soliton-pair rigidly rotated around the $y$ axis, pump and signal fuse into a single beam with (a) $t_{d}>0$ (+6 mrad) and (b) $t_{d}<0$ (-6 mrad). During the buildup of the nonlinear response, the originally crossing and diffracting beams at $t=0$ fuse together at $t=3 \mathrm{~min}$. 
(a)
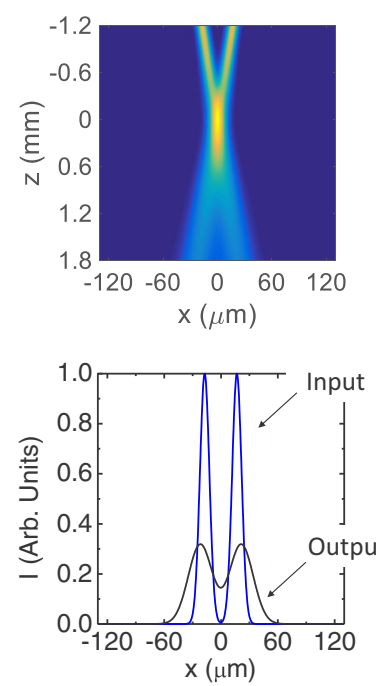

(e)
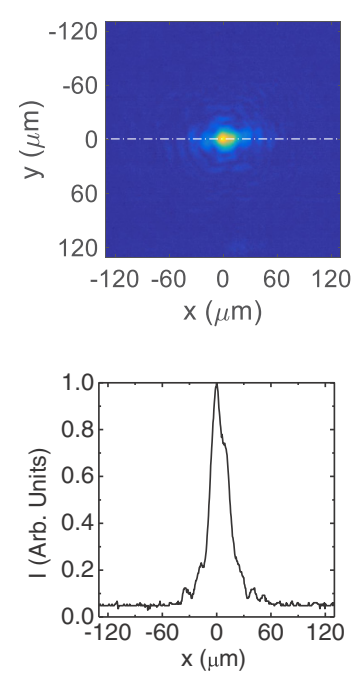

(i)

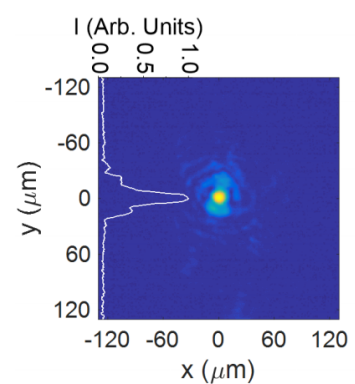

(b)
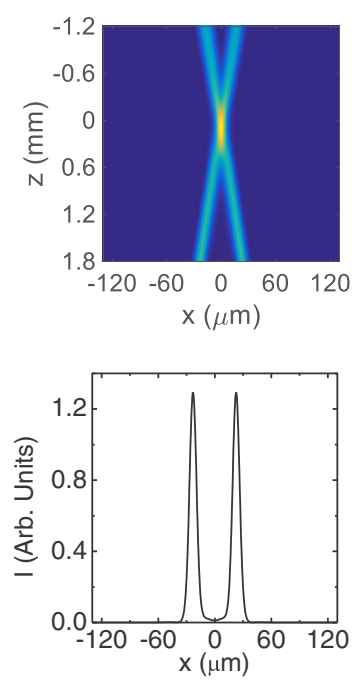

(f)
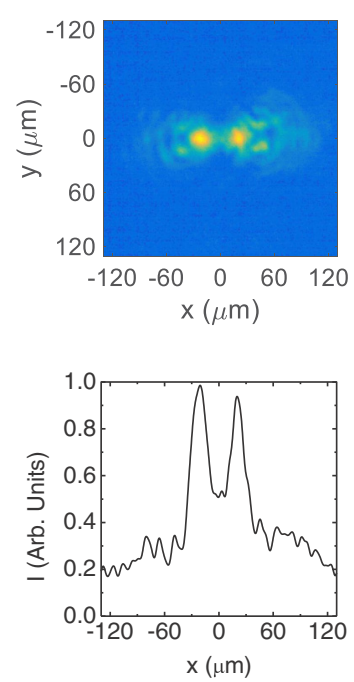

(j)

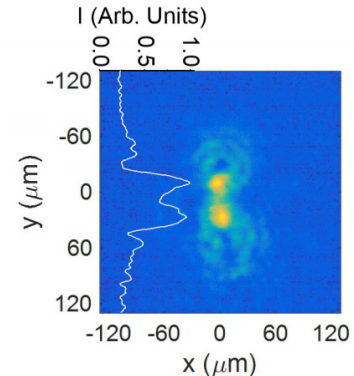

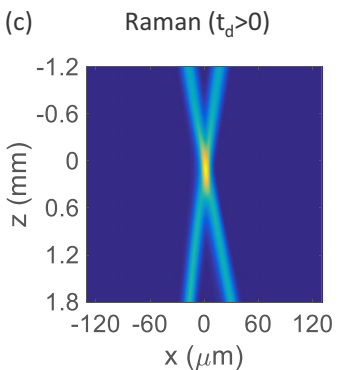

(d) $\operatorname{Raman}\left(\mathrm{t}_{\mathrm{d}}<0\right)$
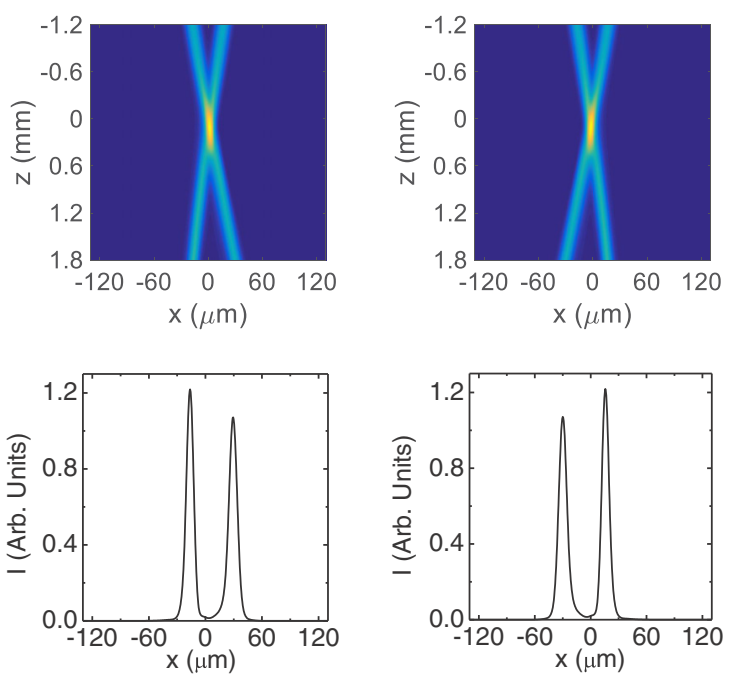

(g) $\quad \operatorname{Raman}\left(\mathrm{t}_{\mathrm{d}}>0\right)$

(h) Raman $\left(t_{d}<0\right)$
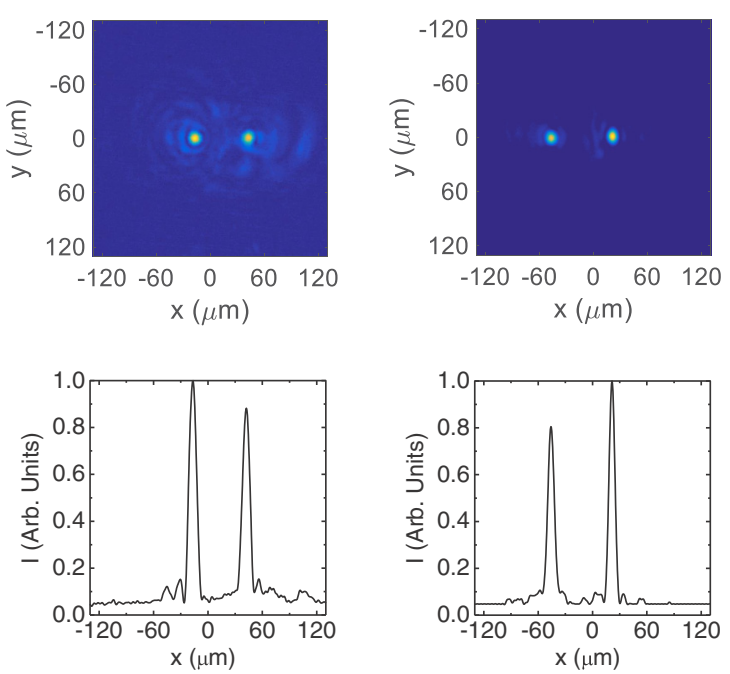

(k)

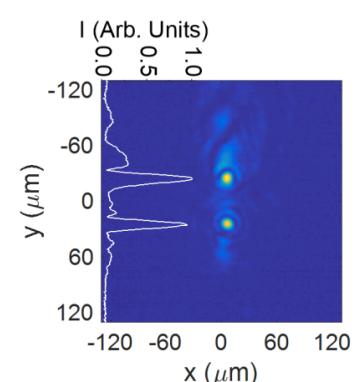

(l)

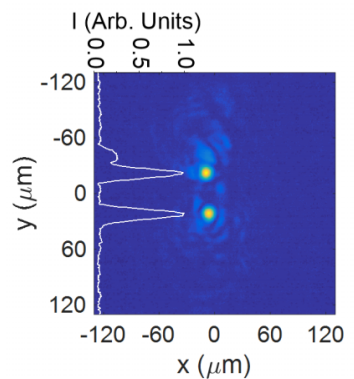

FIG. 6. Incoherent soliton collisions. (a)-(d) Numerical 1 + 1D simulation of collisions between mutually incoherent solitons. (a) Linear propagation intensity distribution in the $x z$ plane (top) and input/output $x$ profiles (bottom) compared to (b) Kerr, (c) Raman with $t_{d}>0$, and (d) Raman with $t_{d}<0$ models. (e)-(h) TWM experiments with mutually incoherent signal and pump solitons. (e) Input intensity distribution (top) and $x$ profile (bottom), (f) linear output, (g) and (h), nonlinear output for $t_{d}>0$ and $t_{d}<0$, respectively. (i)-(l) Incoherent soliton collisions in the $y z$ plane, as for (e)-(h) but with $y$ profiles superimposed on the intensity distribution. 
Eq. (5), for $L_{i}=420 \mu \mathrm{m}, L_{z}=1.8 \mathrm{~mm}$, giving $\alpha=2 \mathrm{~cm}^{-1}$ and a giant soliton gain coefficient $\gamma=80 \mathrm{~cm}^{-1}$. The same experiment can be carried out with different collision angles $\theta$, even if the available angles are limited in our scheme by the numerical aperture of the focusing lens $(\mathrm{NA}=0.17)$. For comparison, in Fig. 3(e) we include selected measurements with $\theta=9 \mathrm{mrad}$ (green triangle) and $15 \mathrm{mrad}$ (red dot), while in the inset of Fig. 3(e) we compare the output soliton profiles for $\theta=12 \mathrm{mrad}$ (black line) in Figs. 3(c) and 3(d) $(m=1)$ to the results for $\theta=9 \mathrm{mrad}$ (green dashed line) and $15 \mathrm{mrad}$ (red dashed line). Results indicate the direction of nonreciprocal energy exchange $(g>1)$ is irrespective of $m$, so that on the sole basis of the sign of $t_{d}$ (i.e., the direction of the external bias field), energy is transferred to the signal even when the signal itself is more intense than the pump. In Fig. 3(f), we compare the values of TWM gain coefficient $g$ versus transverse grating period $\Lambda$ for $m=1$ in the three measured cases of $\theta=9$ (green triangle), 12 (black square), and 15 (red dot) mrad to the values predicted through numerical simulations (as in Fig. 1) (white squares). Even though the model is $1+1 \mathrm{D}$ while the experiment is $2+1 \mathrm{D}$, and the TWM grating period is comparable to the transverse extent of the soliton collision [see the limited number of fringes in Figs. 1(c) and 1(d)], a good agreement is found.

In Fig. 4, we report results for a collision that occurs in the $y z$ plane, orthogonal to the $x$-directed bias field. Since the geometry does not physically distinguish the signal and pump solitons, no TWM is observed. The comparison of collision in the $x z$ and $x y$ planes also indicates that the anisotropy that naturally accompanies two-dimensional PR solitons plays a negligible role in our experiments (see Appendix) [43].

In Fig. 5, we report the observation of reciprocal soliton fusion, i.e., the formation of a single output beam irrespective of the sign of $t_{d}$. The passage from a nonreciprocal to a reciprocal coherent interaction is achieved by matching the asymmetry introduced by the orientation of the external bias field along the $x$ direction with a second asymmetry, achieved by shifting in the $x$ direction the two transmitted spots on the SLM, thus rigidly rotating the two colliding beams around the $y$ axis inside the sample.

\section{CONCLUSIONS}

Nonlinear waves can bounce off each other, spiral, and even fuse together. This leads to a rich wave phenomenology that includes counterintuitive effects such as optical turbulence [44] and wave condensation [45]. A basic hereto unsolved puzzle of nonlinear wave dynamics is the emergence of RWs that in some systems form from a mixture of wave interaction, noise, and extreme nonlinear response, where dynamics are dominated by solitons [46-50]. Our investigation indicates that in conditions where RWs form, soliton-soliton collisions are dominated by a nonreciprocal energy exchange with a giant TWM gain up to $80 \mathrm{~cm}^{-1}$. This provides a microscopic rectification mechanism, an asymmetric amplification, that can then explain out-of-equilibrium statistics not only in optics, but also in other wave systems such as sound and water waves.

\section{ACKNOWLEDGMENTS}

We acknowledge support from the Lazio Innova 2018 Project (No. F83G17000850007), Sapienza-Ricerca di Ateneo 2017 and 2018 Projects (No. RM11715C3FA0E680, No. RG1181643688C4C1), the H2020 Fet project PhoQus, the Doctoral Foundation of Tianjin Normal University (Grant No. 135202XB1607), and the Research and Visiting Abroad Project for Young Scholars of Tianjin Normal University 2015-2020.

\section{APPENDIX}

For each numerical and experimental result reported in the main text, we carried out a comparative simulation and experiment using mutually incoherent pump and signal solitons. This allows us to identify the role of soliton TWM mediated by the nonlocal Raman-like mechanism, which can only intervene in the coherent case, and the role of conventional soliton-soliton energy exchange resulting from nonintegrable corrections to the Kerr nonlinearity. Results are summarized in Fig. 6.
[1] J. P. Bouchaud, Weak ergodicity breaking and aging in disordered systems, J. Phys. I France 2, 1705 (1992).

[2] A. Clauset, C. R. Shalizi, and M. E. J. Newman, Power-law distributions in empirical data, Siam Rev. 51, 661 (2009).

[3] R. P. Feynman, R. Leighton, and M. Sands, The Feynman Lectures on Physics I (Addison-Wesley, Reading, 1963), Chap. 46.

[4] R. D. Astumian, Thermodynamics and kinetics of a Brownian motor, Science 276, 917 (1997).

[5] D. R. Solli, C. Ropers P. Koonath, and B. Jalali, Optical rogue waves, Nature 450, 1054 (2007).

[6] M. Onorato, S. Residori, U. Bortolozzoc, A. Montina, and F. T. Arecchi, Rogue waves and their generating mechanisms in different physical contexts, Phys. Rep. 528, 47 (2013).

[7] J. M. Dudley, F. Dias, M. Erkintalo, and G. Genty, Instabilities, breathers and rogue waves in optics, Nat. Photon. 8, 755 (2014).
[8] S. Birkholz, C. Brée, A. Demircan, and G. Steinmeyer, Predictability of Rogue Events, Phys. Rev. Lett. 114, 213901 (2015).

[9] P. Walczak, S. Randoux, and P. Suret, Optical Rogue Waves in Integrable Turbulence, Phys. Rev. Lett. 114, 143903 (2015).

[10] N. Akhmediev, B. Kibler, F. Baronio, M. Belić, W. Zhong, Y. Zhang, W. Chang, J. M Soto-Crespo, P. Vouzas, P. Grelu, C. Lecaplain, K Hammani, S Rica, A Picozzi, M. Tlidi, K. Panajotov, A. Mussot, A. Bendahmane, P. Szriftgiser, G. Genty, J. Dudley, A. Kudlinski, A. Demircan, U. Morgner, S. Amiraranashvili, C. Bree, G. Steinmeyer, C. Masoller, N. G. R. Broderick, A. F. J. Runge, M. Erkintalo, S. Residori, U. Bortolozzo, F. T. Arecchi, S. Wabnitz, C. G. Tiofack, S. Coulibaly, and M. Taki, Roadmap on optical rogue waves and extreme events, J. Opt. 18, 063001 (2016). 
[11] P. Suret, R. E. Koussaifi, A. Tikan, C. Evain, S. Randoux, C. Szwaj, and S. Bielawski, Single-shot observation of optical rogue waves in integrable turbulence using time microscopy, Nat. Commun. 713136 (2016).

[12] Z. Yang, W. Zhong, M. Belić, and Y. Zhang, Controllable optical rogue waves via nonlinearity management, Opt. Express 26, 7587 (2018).

[13] A. Armaroli, C. Conti, and F. Biancalana, Rogue solitons in optical fibers: A dynamical process in a complex energy landscape, Optica 2, 497 (2015).

[14] D. Pierangeli, F. Di Mei, C. Conti, A. J. Agranat, and E. DelRe, Spatial Rogue Waves in Photorefractive Ferroelectrics, Phys. Rev. Lett. 115, 093901 (2015).

[15] J. M. Soto-Crespo, N. Devine, and N. Akhmediev, Integrable Turbulence and Rogue Waves: Breathers or Solitons, Phys. Rev. Lett. 116, 103901 (2016).

[16] T. Kanna, M. Lakshmanan, P. T. Dinda, and N. Akhmediev, Soliton collisions with shape change by intensity redistribution in mixed coupled nonlinear Schrödinger equations, Phys. Rev. E 73, 026604 (2006).

[17] R. Driben and I. Babushkin, Accelerated rogue waves generated by soliton fusion at the advanced stage of supercontinuum formation in photonic-crystal fibers, Opt. Lett. 37, 5157 (2012).

[18] A. V. Yulin, R. Driben, B. A. Malomed, and D. V. Skryabin, Soliton interaction mediated by cascaded four wave mixing with dispersive waves, Opt. Express 21, 14481 (2013).

[19] P. A. Andrekson, N. A. Olsson, P. C. Becker, J. R. Simpson, T. TanbunEk, R. A. Logan, and K. W. Wecht, Observation of multiple wavelength soliton collisions in optical systems with fiber amplifiers, Appl. Phys. Lett. 57, 1715 (1990).

[20] K. Kurokawa, H. Kubota and M. Nakazawa, Soliiton selffrequency shift accelerated by femtosecond soliton interaction, Electron. Lett. 28, 2052 (1992).

[21] A. Mussot, A. Kudlinski, M. Kolobov, E. Louvergneaux, M. Douay, and M. Taki, Observation of extreme temporal events in CW-pumped supercontinuum, Opt. Express 17, 17010 (2009).

[22] J. P. Gordon, Theory of the soliton self-frequency shift, Opt. Lett. 11, 662 (1986).

[23] Y. Kodama and K. Nozaki, Soliton interaction in optical fibers, Opt. Lett. 12, 1038 (1987).

[24] B. J. Hong and C. C. Yang, Interactions between femtosecond solitons in optical fibers, J. Opt. Soc. Am. B 8, 1114 (1991).

[25] G. Agrawal, Nonlinear Fiber Optics, 3rd ed. (Academic, San Diego, 2001).

[26] P. Yeh, Two-wave mixing in nonlinear media, IEEE J. Quantum Electron. 25, 484 (1989).

[27] D. Timotijević, M. Belić, and R. W. Boyd, Two- and four-wave mixing with saturable absorption and gain, IEEE J. Quantum Electron. 28, 1915 (1992).

[28] N. Akhmediev, W. Królikowski, A. J. Lowery, Influence of the Raman-effect on solitons in optical fibers, Opt. Commun. 131, 260 (1996).

[29] F. Luan, D. V. Skryabin, A. V. Yulin, J. C. Knight, Energy exchange between colliding solitons in photonic crystal fibers, Opt. Express 14, 9844 (2006).
[30] S. Chi and S. Wen, Raman cross talk of soliton collision in a lossless fiber, Opt. Lett. 14, 1216 (1989).

[31] Y. C. Chang, C. Wang, S. Yin, R. C. Hofman, and A. G. Mott, Giant, electro-optic effect in nanodisordered KTN crystals, Opt. Lett. 38, 4574 (2013).

[32] D. Pierangeli, F. Di Mei, J. Parravicini, GB. Parravicini, A. J. Agranat, C. Conti, and E. DelRe, Observation of an intrinsic nonlinearity in the electrooptic response of freezing relaxors ferroelectrics, Opt. Mater. Express 4, 1487 (2014).

[33] D. Pierangeli, G. Musarra, F. Di Mei, G. Di Domenico, A. J. Agranat, C. Conti, and E. DelRe, Enhancing optical extreme events through input wave disorder, Phys. Rev. A 94, 063833 (2016).

[34] D. Pierangeli, F. Di Mei, G. Di Domenico, A. J. Agranat, C. Conti, and E. DelRe, Turbulent Transitions in Optical Wave Propagation, Phys. Rev. Lett. 117, 183902 (2016).

[35] E. DelRe, A. D’Ercole, and E. Palange, Mechanisms supporting long propagation regimes of photorefractive solitons, Phys. Rev. E. 71, 036610 (2005).

[36] E. DelRe, B. Crosignani, and P. Di Porto, Prog. Opt. 53, 153 (2009).

[37] The term plays a role only when the sample is unbiased $\left(E_{0}=\right.$ 0 ) and operated exactly at the Curie point $T=T_{C}$, see, for example, E. DelRe, F. Di Mei, J. Parravicini, G. Parravicini, A. J. Agranat, and C. Conti, Subwavelength anti-diffracting beams propagating over more than 1000 Rayleigh lengths, Nat. Photon. 9, 228 (2015).

[38] M. Chi, J. Huignard, and P. M. Petersen, A general theory of two-wave mixing in nonlinear media, J. Opt. Soc. Am. B 26, 1578 (2009).

[39] W. Królikowski, and S. A. Holmstrom, Fusion and birth of spatial solitons upon collision, Opt. Lett. 22, 369 (1997).

[40] P. Yeh, Introduction to Photorefractive Nonlinear Optics, Wiley Series in Pure and Applied Optics (John Wiley and Sons, Inc., New York, 1993).

[41] W. Królikowski, N. Akhmediev, B. Luther-Davies, and M. Cronin-Golomb, Self-bending photorefractive solitons, Phys. Rev. E. 54, 5761 (1996).

[42] M. H. Frosz, O. Bang, and A. Bjarklev, Soliton collision and Raman gain regimes in continuous-wave pumped supercontinuum generation, Opt. Express 14, 9391 (2006).

[43] E. DelRe, A. Ciattoni, and A. J. Agranat, Anisotropic charge displacement supporting isolated photorefractive optical needles, Opt. Lett. 26, 908 (2001).

[44] A. Picozzi, J. Garnier, T. Hansson, P. Suret, S. Randoux, G. Millot, and D. N. Christodoulides, Optical wave turbulence: Towards a unified nonequilibrium thermodynamic formulation of statistical nonlinear optics, Phys. Rep. 542, 1 (2014).

[45] U. Bortolozzo, J. Laurie, S. Nazarenko, and S. Residori, Optical wave turbulence and the condensation of light, J. Opt. Soc. Am. B 26, 2280 (2009).

[46] Y. S. Kivshar, and G. Agrawal, Optical Solitons: From Fibers to Photonic Crystals (Elsevier, San Diego, 2003).

[47] B. A. Malomed, D. Mihalache, F. Wise, and L. Torner, Spatiotemporal optical solitons, J. Opt. B: Quantum Semiclass. Opt. 7, R53 (2005). 
[48] S. Birkholz, E. T. J. Nibbering, C. Brée, S. Skupin, A. Demircan, G. Genty, and G. Steinmeyer, Spatiotemporal Rogue Events in Optical Multiple Filamentation, Phys. Rev. Lett. 111, 243903 (2013).

[49] M. Wimmer, A. Regensburger, M.-A. Miri, C. Berch, D. N. Christodoulides, and U. Peschel, Observation of optical solitons in PT-symmetric lattices, Nat. Commun. 6, 7782 (2015).

[50] J. Peng, N. Tarasov, S. Sugavanam, and D. Churkin, Rogue waves generation via nonlinear soliton collision in multiplesoliton state of a mode-locked fiber laser, Opt. Express 24, 21256 (2016) 